\title{
Plädoyer für eine Abkehr von der glukosezentrierten Diabetestherapie
}

\author{
Bei der Behandlung von Typ-1-Diabetikern sollte der Surrogatparameter HbA $\mathrm{Ac}_{c}$ nicht zu sehr im \\ Mittelpunkt stehen, so Dr. Stefan Kopf aus Heidelberg in Berlin. Er plädierte für eine individuali- \\ sierte multimodale Therapie, die sich an den Risikofaktoren der Patienten orientiert.
}

Die Empfehlung einer strikten Blutzuckersenkung bei Typ-1Diabetikern mit dem $\mathrm{HbA}_{1 \mathrm{c}}$-Ziel meist $<7,5 \%$ basiert gerade einmal auf Daten von rund 1800 Patienten, im wesentlichen Teilnehmern der DCCT-Studie, betonte Kopf, Oberarzt an der Studienambulanz für Diabetes-Forschung des Uniklinikums Heidelberg. Die in der Studie durch eine intensivierte Insulintherapie $\left(\mathrm{HbA}_{1 \mathrm{c}}\right.$-Senkung von $8 \%$ auf $7 \%$ vs. konventionelle Therapie) erzielten deutlichen relativen Risikoreduktionen seien nicht mehr so beeindrucken, wenn das absolute Risiko für Makro- und Mikrokomplikationen miteinander verglichen wird. Beispiel terminale Niereninsuffizienz (ESRD): Relativ wurde das ERSD-Risiko um rund 50\% gesenkt (8 vs. 16 Fälle), absolut war es im Verlauf von 16 Jahren aber nur um 1,35\% verringert. Also: 74 Patienten mussten 16 Jahre intensiv therapiert werden, um 1 ESRD zu verhindern $(\mathrm{NNT}=74)$. Bei der diabetische Neuropathie: relative Risikoreduktion $21 \%$, absolute 6\%. 17 Patienten müssten über 13-14 Jahre intensiv therapiert werden, um 1 Ereignis zu verhindern.

Laut den 30-Jahres-Langzeitdaten der DCCT-Studie war die Sterberate durch kardiovaskuläre Ereignisse im Intensivarm relativ um 30\% verringert (9 vs. 16 Fälle), das absolute Risiko unterschied sich aber nur um 2,3\%. Die NNT über 30 Jahre für einen kardiovaskulären Tod betrug 108. Nur 11\% aller Spätschäden in DDCT seien durch die Diabetesdauer und den $\mathrm{HbA}_{1 \mathrm{c}}$ zu erklären, betonte Kopf. Andererseits korrelierte die intensivierte Therapie mit mehr schweren Hypoglykämien und Koma (20,9\% vs. $15,6 \%)$ sowie einer erhöhten Unfall- und Suizidrate (30,2\% vs. 7,8\%). „Wir sollten Typ-1-Diabetiker nicht glukosezentrisch, sondern individuell behandeln", so der Diabetologe. Und für diese doch einfache Botschaft erhielt der Referent viel Beifall seiner Zuhörer. „Danke, dass Sie mir für meine übliche Versorgung der Patienten in der Praxis so viel Unterstützung geliefert haben", sagte ein niedergelassener Diabetologe aus dem Auditorium und ergänzte: „So eine kritische Betrachtung der Blutzuckereinstellung habe ich auf einem DDG-Kongress noch nie gehört.”

\section{Wie sollte also konkret vorgegangen werden?}

Die Patienten sollten nach Risikofaktoren und dem Gesamtbild, u.a. Ernährungzustand, in Subgruppen eingeteilt werden, riet Kopf. Eine multimodale Diabetestherapie sollte auf eine gute Einstellung von Blutdruck, Lipiden und den Erhalt der Nierenfunktion zielen und nicht nur den $\mathrm{HbA}_{1 \mathrm{c}}$, „Es ist nur ein Surrogatmarker", betonte der Diabetologe. Keiner könne das optimale Blutzucker Tagesprofil nennen. Habe ein Patienten schon eine schwere Hypoglykämie gehabt, sollte der $\mathrm{HbA}_{1 \mathrm{c}}$ angehoben werden.

Zwar ist die Lebenserwartung von Typ-1-Diabetikern im Schnitt immer noch um fast zehn Jahre kürzer als von Personen ohne Störung des Glukosemetabolismus. Aber mit zunehmendem Alter werden die Unterschiede geringer. Senioren mit Typ1-Diabetes haben praktisch die gleiche Lebenserwartung wie Stoffwechselgesunde, so Prof. Thomas Haak, Bad Mergentheim.

Die Blutzuckereinstellung ohne Hypoglykämien zählt bei Typ1- ebenso wie bei Typ-2-Patienten zu den Grundsätzen einer modernen Diabetestherapie. Bei älteren Patienten mit jahrzehntelanger Diabetesdauer ist das Risiko für schwere Hypoglykämien besonders hoch. Zudem erholen sich die Patienten in der Regel auch langsamer von einer Hypoglykämie als jüngere Patienten, sagte Prof. Andreas Fritsche aus Tübingen. Ein besonders großes Problem: Rund ein Viertel aller Typ-1-Patienten leidet unter einer Hypoglykämie-Wahrnehmungsstörung, bei der die gegenregulatorische Hormonantwort während einer Hypoglykämie reduziert ist. Es fehlen daher die ersten Warnsymptome wie Schwitzen, die auf einen gefährlichen Abfall der Blutglukose hinweisen. Bei Patienten mit langer Diabetesdauer liegt der Anteil derer, die pro Jahr eine schwere Hypoglykämie erleiden, bei bis zu 50\%, sagte Fritsche. Der Diabetologe empfahl zur Vorbeugung regelmäßige Schulungen von Typ-1-Diabetikern, am besten in der Wochenklinik. Solche Schulungen trügen auch zur Verbesserung der Blutzuckerwahrnehmung bei.

Roland Fath

Quelle: Symposium: „Typ-1-Diabetes im mittleren und höheren Lebensalter", 51. DDG-Jahrestagung, 04.-07.05.2016, Berlin

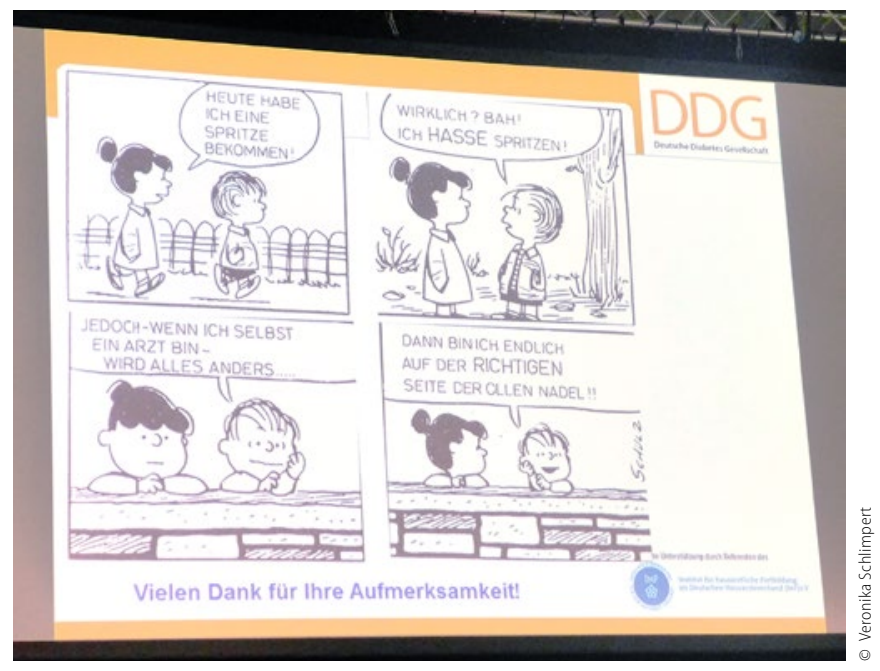

Auch der Spaßfaktor kam beim ein oder anderen Vortrag des 51. DDG-Jahreskongresses nicht zu kurz. 